\title{
Developing High Brightness Beams for Heavy lon Driven Inertial Fusion
}

\author{
J. W. Kwan ${ }^{\mathrm{a}}$, L.A. Ahle ${ }^{\mathrm{b}}$, A. Anders ${ }^{\mathrm{a}}$, F. M. Bieniosek ${ }^{\mathrm{a}}$, E. Chacon-Golcher ${ }^{\mathrm{a}}$, D. P. \\ Grote $^{\mathrm{b}}$, E. Henestroza ${ }^{\mathrm{a}}$, K.N. Leung ${ }^{\mathrm{a}}$, A.W. Molvik ${ }^{\mathrm{b}}$ \\ ${ }^{\mathrm{a}}$ Lawrence Berkeley National Laboratory, Berkeley, CA \\ ${ }^{\mathrm{b}}$ Lawrence Livermore National Laboratory, Livermore, CA
}

\begin{abstract}
Heavy ion fusion (HIF) drivers require large currents and bright beams. In this paper we review the two different approaches for building HIF injectors and the corresponding ion source requirements. The traditional approach uses large aperture, low current density ion sources, resulting in a very large injector system. A more recent conceptual approach merges high current density mini-beamlets into a large current beam in order to significantly reduce the size of the injector. Experiments are being prepared to demonstrate the feasibility of this new approach.
\end{abstract}

Paper to be submitted to the $9^{\text {th }}$ International Conference on Ion Sources, Oakland, CA Sept. 3-7, 2001 


\section{INTRODUCTION}

Heavy ion driven inertial fusion (HIF) requires about 3-7 MJ to achieve ignition with a D-T target [1]. Recent target designs assume $\mathrm{Pb}^{+}$ions at $2-4 \mathrm{GeV}$; but lighter ions with lower energy, that give the same range in the target, would give equivalent results and lead to a lower cost power plant [2]. Along with reduced cost comes the increased technical difficulty of injecting higher charge than the $\sim 1 \mathrm{mC}$ required for $\mathrm{Pb}^{+}$, and the greater degree of beam charge neutralization required for focusing the beams of higher space charge onto the target in the fusion chamber.

Induction linacs can accelerate and compress these beams from $\sim 10 \mu$ s at injection to $\sim 300 \mathrm{~ns}$ by the end of the driver and $\sim 10 \mathrm{~ns}$ at the target. The total beam current from the ion source is $\sim 50-100 \mathrm{~A}$ (more for lighter ions). In order to overcome the space charge problem associated with high current heavy ion beams, an HIF driver is usually designed to contain an array of $\mathrm{N} \sim 100$ parallel ion beam channels at $\sim 0.5 \mathrm{~A}$ each.

In order to focus the ion beams onto a mm-size fusion target the beam emittance must be small, thus HIF requires beams with both large current and high brightness. In terms of ion source parameters, the beam brightness is proportional to $\mathrm{J} / \mathrm{T}$ where $\mathrm{J}$ is the current density and $\mathrm{T}$ is the effective ion temperature. So high brightness demands either high current density and/or low ion temperature. Furthermore, a heavy ion injector must have an adequate low energy beam transport (LEBT) system that can handle the severe space charge force. The LEBT can limit the maximum current density in the injector and often impose restrictions on the ion source.

Although heavy ions are ultimately needed for fusion drivers, lighter ions such as $\mathrm{K}^{+}$and $\mathrm{Ar}^{+}$can be useful in the near future because they provide an opportunity to do experiments at high ion velocities on medium length accelerator facilities during the early development phases.

\section{LARGE APERTURE METHOD}

According to high voltage breakdown and Child-Langmuir space-charge flow scalings, the current density of a diode decreases as the extracted beam current increases [3]. Thus producing large current and high brightness from a single large aperture implies low ion temperature because the current density must be low. This is the traditional approach to HIF injector. Ions are extracted by a diode (a few hundred $\mathrm{kV}$ ) followed by an ESQ accelerator and a long matching section to compress the beam size and transform a round beam from an ion gun into an elliptical beam for an ESQ lattice [4].

A preliminary design of a multiple beam injector based on the traditional approach was reported by Kwan et al [5]. Figure 1 is a schematic diagram of the outermost beamline in an array of 84 beamlines each carrying about $0.5 \mathrm{~A}$ of beam current. Computer simulation of the ion gun design is shown in figure 2. In this method, the ion source end of the injector is much larger in area than the exit end, so a multiple-beam injector system will have a funnel shape. Beams in the outer layer must be gradually steered towards the axis using long ESQ channels in the matching section. This injector design is not considered attractive because of its large size (and cost). Of course, this 
approach may still be useful for near-term experiments requiring only a single-beam injector without steering.

\section{MINI-BEAMLETS METHOD}

A second method is being explored to construct HIF injectors by maximizing the current density to achieve high brightness. Such a system starts with an array of high current density mini-beamlets (of a few $\mathrm{mA}$ each) that are kept separated from each other within a set of acceleration grids in order to overcome the space charge expansion. After they have gained sufficient kinetic energy $(>1.2 \mathrm{MeV})$, the mini-beamlets will be allowed to merge together to form a high current beam $(\approx 0.5 \mathrm{~A})$. The final beam brightness depends on the emittance of the merged beam. Figure 3 is a schematic diagram of an injector beamline based on this concept. The total length from the ion source to the end of merging/matching section is expected to be only about $1.0 \mathrm{~m}$. With a high enough average current density, the injector transverse dimension can stay constant, so no steering is required in the ESQ channels. Comparing figures 1 and 3, we found a factor of six reduction in size using the new approach.

The main physics issues involved in the mini-beamlets approach are emittance growth and envelope matching in the merging process. To minimize the emittance growth, it is necessary to closely pack a large number of high current density minibeamlets. Simulation has confirmed that the final emittance depends only weakly on the initial emittance of the beamlets because the merging process dominates the emittance growth. This also means that ion sources with higher ion temperature can be used as long as the average current density remains high (using high transparency grids). Figure 4 is an Einzel system designed to accelerate a $2 \mathrm{~mm}$ diameter $\mathrm{K}^{+}$beamlet, with a current density of $100 \mathrm{~mA} / \mathrm{cm}^{2}$, to $1.2 \mathrm{MeV}$ kinetic energy. The beamlets are arranged to fill an elliptical pattern and are aimed differently in the two transverse planes in such a way that when merged, the final beam spot is an ellipse matching the entrance requirements of an ESQ channel [6].

In order to obtain high average beam brightness, it is necessary to develop high transparency accelerator grids operating at high voltage gradient to focus the beamlets. The engineering issues are similar to, but in some way more stringent than, that of neutral beam injectors for magnetic fusion devices. A similar experiment that merged $19 \mathrm{H}^{-}$ beamlets into an ESQ channel had been done before (as part of the neutral beam injector development for ITER) [7,8]. but the HIF injector will involve a lot more beamlets and at much higher current and brightness.

\section{HIF ION SOURCE SPECIFICATIONS AND TYPES}

Aside from the beam current, pulse length, and emittance mentioned above, there are other important parameters for HIF ion sources. Table 1 is a summary of the approximate specifications. A short rise time is important in order to minimize the beam front current and energy variation. Significant energy spread (related to longitudinal 
emittance) will produce a chromatic aberration at the final focussing lens system. In principle, higher charge state $(Z>1)$ ions can reduce the accelerator length but require higher beam current. As far as the driver is concerned, heavy ions of higher charge state are similar to lighter ions with the same charge to mass ratio. However, it is important not to have a mixture of charge states in the linac.

Negative ions are also interesting candidates. HIF beam transport studies have shown that a large positive beam potential (many $\mathrm{kV}$ ) can trap excessive secondary electrons, thereby affecting the focussing. In principle, this can be avoided by using negative ion beams. However, negative ions are generally difficult to produce and they have a large cross-section for electron detachment, leading to significant beam loss in the accelerator.

Table 1. HIF driver ion source and injector specifications

\begin{tabular}{ll}
\hline Beam energy $(\mathrm{MeV})$ & $1.6-2.0$ \\
Beam current per beam channel $(\mathrm{A})$ & 0.5 \\
Beam pulse width $(\mu \mathrm{s})$ & 20 \\
Beam rise-time (fraction of pulse width) & $\leq 5 \%$ \\
Repetition rate $(\mathrm{Hz})$ & 10 \\
Ion mass $(\mathrm{AMU})$ & $84-238$ \\
Charge state purity & $>90 \%$ \\
Emittance $(\pi-\mathrm{mm}-\mathrm{mrad}$, normalized $)$ & $<1.0$ \\
Brightness $=\mathrm{I} /\left(\pi \varepsilon_{\mathrm{x}} \varepsilon_{\mathrm{y}}\right)\left(\mathrm{A} /(\pi \text {-mm-mrad })^{2}\right)$ & $>0.16$ \\
Current fluctuation and pulse-to-pulse variation & $\leq 1 \%$ \\
Current profile uniformity & $>95 \%$ \\
Beam energy spread $(\mathrm{kV})$ & $\leq 2$ \\
Lifetime (pulses) & 108 \\
\hline
\end{tabular}

Since the large aperture method requires an ion source that has a rigid emitter surface and ion temperature below an $\mathrm{eV}$, the only known solution is the surface ionization source. This type of ion source can produce singly ionized alkali metal ions. The tungsten substrate must be kept at $\approx 1050^{\circ} \mathrm{C}$ continuously even though the beam duty factor is $<2 \times 10^{-4}$. This means poor power efficiency and limited lifetime on the heater filaments. For long term, steady state application, the ionizer must be replenished with alkali vapor. The accumulation of neutral alkaline atoms (continuously emitted from the source surface) in the accelerator will eventually degrade the high voltage breakdown threshold.

In principle, a contact ionization source at $>1250^{\circ} \mathrm{C}$ is capable of producing current density $>50 \mathrm{~mA} / \mathrm{cm}^{2}\left(\mathrm{Cs}^{+}\right)$; thus it can be a candidate for the mini-beamlets method if the neutral emission is not too high. However, gas discharge ion sources and arc sources are more effective in producing very high current density beams with excellent power efficiency. The ion temperatures for these sources are higher, but that is an acceptable condition for the mini-beamlet approach. 
Previously, Ahle et al [9] reported testing of a $10 \mathrm{~cm}$ diameter rf-driven multicusp source for HIF application. The results showed that $100 \mathrm{~mA} / \mathrm{cm}^{2}$ of argon ions can be achieved with $3 \mathrm{~kW}$ of rf power. Under certain conditions, the pulse rise-time was optimized to $<2 \mu$ s and the gas pressure in the source $\approx 1 \mathrm{mTorr}$. It is important to operate at low gas pressure because charge exchange can take place in the region below a few hundred $\mathrm{keV}$ where the cross-section is large. Charge exchange produces slower moving ions and therefore increases energy dispersion.

The metal vapor vacuum arc (MEVVA) ion source has the advantages of producing high currents and not requiring gas. However, beam noise and mixed charge states are typical problems in applying vacuum arc ion sources to HIF. Beam noise and mixed charge states are due to the explosive plasma formation mechanism; these issues have been addressed with some success by manipulating the fully ionized plasma with biased meshes, see Oks et al. [10] and Anders et al. [11] presented at this conference, and references therein. The challenge is to simultaneously obtain all parameters as defined by Table 1. The degree of charge state purity and low noise of a vacuum arc depend greatly on the choice of cathode material which is the plasma's feedstock material. Bismuth has been identified as a promising candidate. Further experiments are being planned to improve current density, reduce beam noise, and to measure the beam emittance.

\section{EXPERIMENTAL STATUS}

Recently we have completed the modification of a large-aperture injector for a new experiment. The injector has a $10-\mathrm{cm}$ diameter ionizer producing $>0.5 \mathrm{~A} \mathrm{of} \mathrm{K}^{+}$. Detail results and comparison with simulations can be found in Bieniosek et al [12]. Measurements are also in progress to characterize high current density ionizers and the emission of neutral alkali vapor [13].

At present, the main goal of our research program is to experimentally study the mini-beamlets approach. A new $25 \mathrm{~cm}$ diameter rf-driven multicusp source and a new 20 $\mu \mathrm{s}, 500 \mathrm{kV}$ test stand have been constructed for this purpose. Figure 5 is a schematic diagram of the apparatus to be built for doing experiments with beamlet merging and matching.

\section{CONCLUSION AND ACKNOWLEDGEMENT}

Heavy ion fusion drivers require large current and bright beams that not yet available from today's ion source technology. The traditional method uses large aperture, low current density ion sources but presently we are investigating another method that uses merged high current density beamlets for a more compact injector design. This work is funded by US DOE under contract DE-AC03-76SF00098 (LBNL) and contract W7405-ENG-48 (LLNL).

\section{REFERENCES}

[1] D. A. Callahan-Miller, M. Tabak, Nuclear Fusion 39, 1547 (1999).

[2] W.R. Meier, Fusion Technology 34, 326 (1998). 
[3] J.W. Kwan, Rev. Sci. Instrum, 71(2), p807, (2000).

[4] S. Yu, et al, Proc. of Inter. Symp. on Heavy Ion Inertial Fusion; Fusion Engineering and Design, 32-33, p309, (1996)

[5] J.W. Kwan, et al, Proc. Particle Accelerator Conf., New York, March, (1999), p1937

[6] J.W. Kwan, et al, Nucl. Instrum. and Methods (A), 464, p379, (2001)

[7] T. Inoue, et al., Rev. Sci. Instrum, 66(7), p3859, (1995).

[8] J.W. Kwan, et al, Rev. Sci. Instrum, 66(7), p3864, (1995).

[9] L. Ahle, et al, Proc. Particle Accelerator Conf., Chicago, June, (2001)

[10] E. Oks, G. Yushkov, I. Litovko, A. Anders, and I.G. Brown, Further development of low noise Mevva ion source, these proceedings (2001).

[11] A. Anders and R. Hollinger, Reducing ion beam noise of vacuum arc ion sources by a spcace charge filter, these proceedings (2001).

[12] F. M. Bieniosek, E. Henestroza, J.W. Kwan, Performance of the $\mathrm{K}^{+}$ion diode in the 2-MV Injector for Heavy Ion Fusion, this proceeding, (2001)

[13] E. Chacon-Golcher, D. Baca, J.W. Kwan, Development of High Current Density Surface Ionization Sources for Heavy Ion Fusion Applications, this proceeding, (2001) 


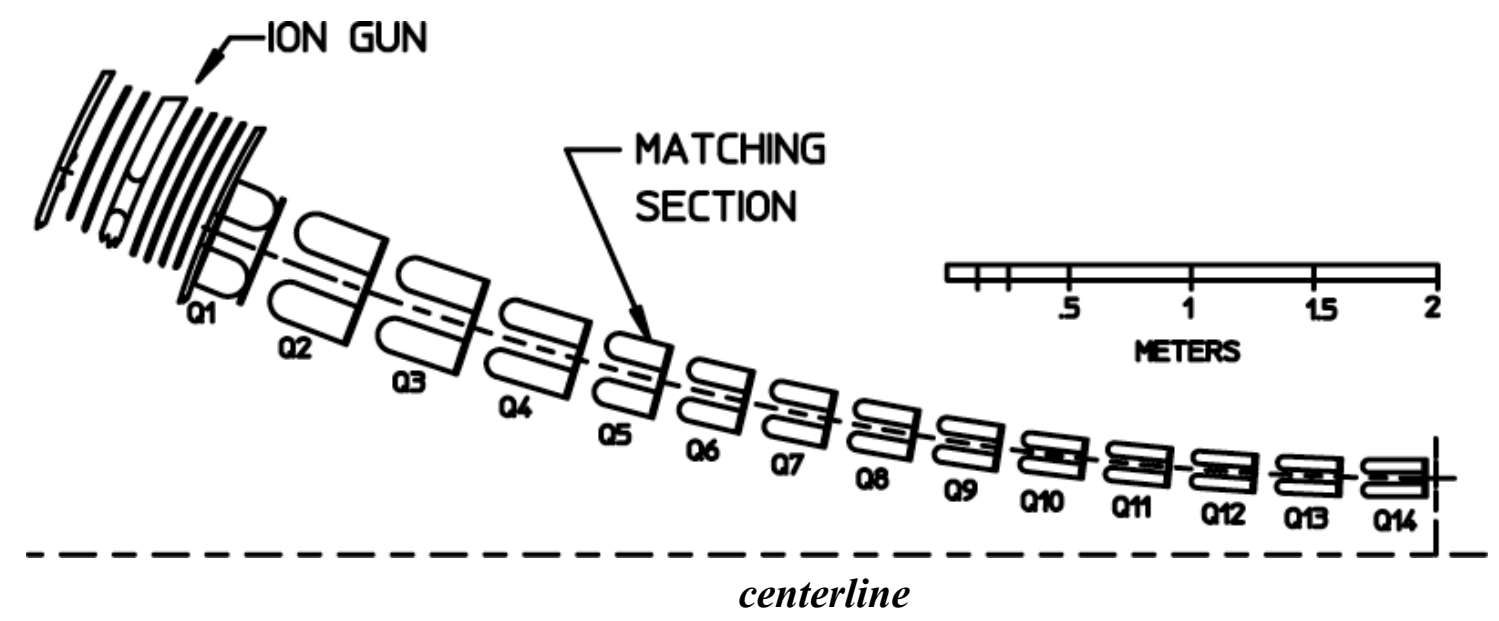

Fig. 1. Schematic diagram of the outer most beamline in the matching section.

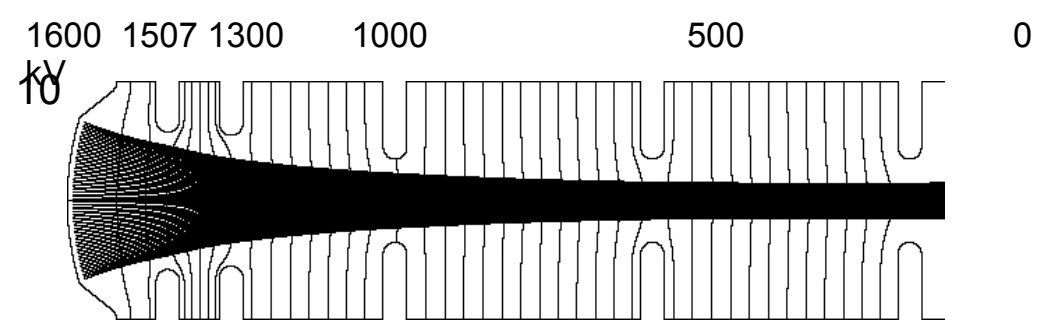

$r(\mathrm{~cm})$

Fig. 2. Ion gun design for a low current density $0.5 \mathrm{~A} \mathrm{~K}^{+}$beam. $-10$

$\begin{array}{llr}20 & 40 & 60 \mathrm{~cm} \\ \text { Z- axis }\end{array}$




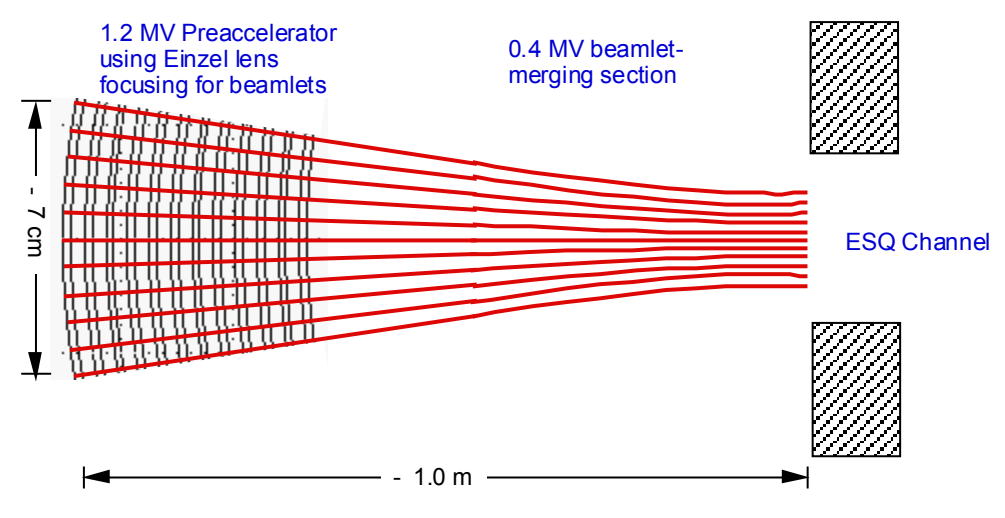

Fig. 3. Schematic diagram of an injector beamline based on merging mini-beamlets.

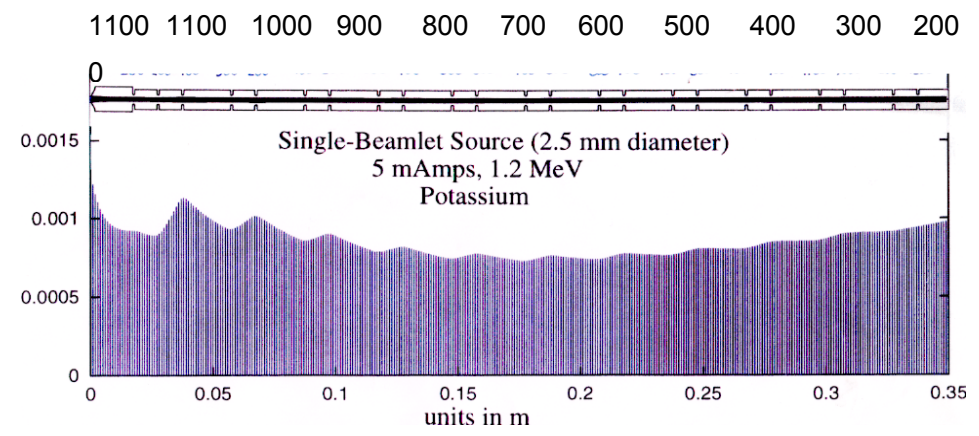

$$
\text { Z (m) }
$$

Fig. 4. An ion gun design for a $5 \mathrm{~mA}, 1.2 \mathrm{MeV} \mathrm{K}^{+}$beamlet. 


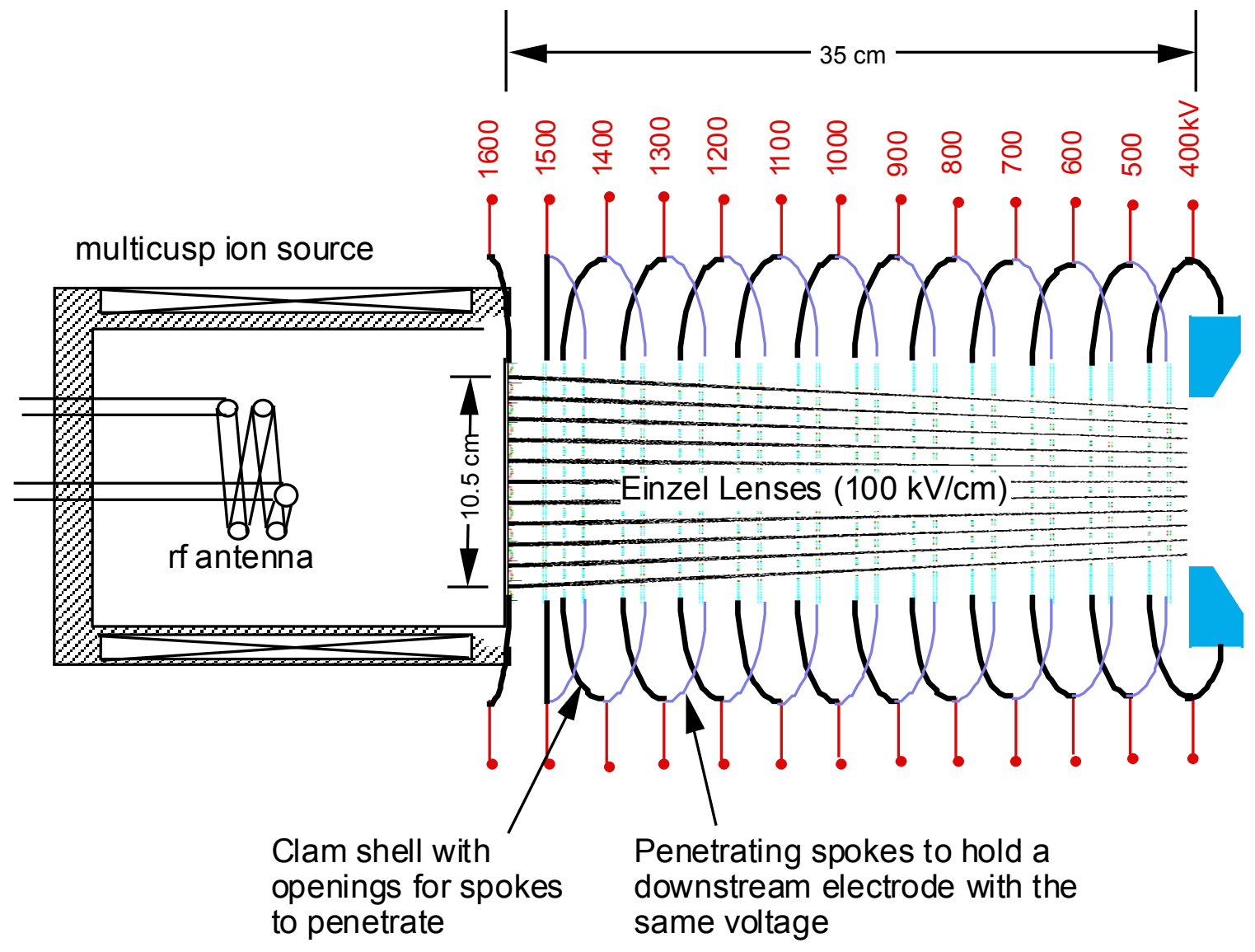

Fig. 5 Schematic diagram of the mini-beamlet pre-accelerator grids fitted with a rf-driven gas source. 